\title{
Nonlinear Oscillatory Shear Tests in Viscoelastic Holography
}

\author{
Matteo Baggioliø* \\ Instituto de Fisica Teorica UAM/CSIC, c/Nicolas Cabrera 13-15, Universidad Autonoma de Madrid, \\ Cantoblanco, 28049 Madrid, Spain
}

Sebastian Grieninger $\odot^{\dagger}$

Theoretisch-Physikalisches Institut, Friedrich-Schiller-Universität Jena, Max-Wien-Platz 1, D-07743 Jena, Germany and Department of Physics, University of Washington, Seattle, Washington 98195-1560, USA

\author{
Hesam Soltanpanahi \\ Guangdong Provincial Key Laboratory of Nuclear Science, Institute of Quantum Matter, \\ South China Normal University, Guangzhou 510006, China;
}

Institute of Theoretical Physics, Jagiellonian University, S. Łojasiewicza 11, PL 30-348 Kraków, and Poland; and School of Physics, Institute for Research in Fundamental Sciences (IPM), P.O. Box 19395-5531, Teheran, Iran

(Received 25 October 2019; revised manuscript received 25 January 2020; accepted 5 February 2020; published 26 February 2020)

\begin{abstract}
We provide the first characterization of the nonlinear and time dependent rheologic response of viscoelastic bottom-up holographic models. More precisely, we perform oscillatory shear tests in holographic massive gravity theories with finite elastic response, focusing on the large amplitude oscillatory shear (LAOS) regime. The characterization of these systems is done using several techniques: (i) the Lissajous figures, (ii) the Fourier analysis of the stress signal, (iii) the Pipkin diagram and (iv) the dependence of the storage and loss moduli on the amplitude of the applied strain. We find substantial evidence for a strong strain stiffening mechanism, typical of hyperelastic materials such as rubbers and complex polymers. This indicates that the holographic models considered are not a good description for rigid metals, where strain stiffening is not commonly observed. Additionally, a crossover between a viscoelastic liquid regime at small graviton mass (compared to the temperature scale), and a viscoelastic solid regime at large values is observed. Finally, we discuss the relevance of our results for soft matter and for the understanding of the widely used homogeneous holographic models with broken translations.
\end{abstract}

DOI: 10.1103/PhysRevLett.124.081601

Introduction.-In elastic solids, the mechanical stress is proportional to the applied external shear strain [1]. However, in hydrodynamic fluids the stress is proportional to the shear rate [2]. Of course, both of these cases are abstract idealizations, valid only under limiting conditions. In general, all the materials are viscoelastic - they present an interplay between elastic effects and dissipative viscous ones [3]; honey is the most common example.

The idea that "everything flows if you wait long enough" lies behind the foundation of a new field of research known as rheology [4,5] - the study of deformation and flow of matter. Even though the first model goes back to Maxwell in 1867 [6], a large part of the theoretical description of viscoelastic materials is still based on phenomenological frameworks (Kelvin-Voigt, generalized Maxwell, Burgers) [7].

Published by the American Physical Society under the terms of the Creative Commons Attribution 4.0 International license. Further distribution of this work must maintain attribution to the author(s) and the published article's title, journal citation, and DOI. Funded by SCOAP ${ }^{3}$.
The fundamental difficulties are twofold: (i) it is conceptually hard to incorporate dissipation into the effective field theory description of solid materials [8,9] because of the unavoidable requirement of unitarity; (ii) elasticity theory can be formulated in the language of standard effective field theory following a well-defined action principle [10]. Hydrodynamics, on the contrary, is usually described by a set of conservation equations and constitutive relations [11] and it is not suitable for a description in terms of a local and Hermitian action [12].

The problem becomes even more acute when the amplitude of the applied external strain is not small and the linear approximation is of no help anymore-the onset of nonlinear viscoelasticity [13-16]. For simplicity, in this Letter, we focus on oscillatory shear tests in which the external shear strain takes a simple sinusoidal form

$$
\gamma(t)=\gamma_{0} \sin (2 \pi \omega t)
$$

where $\gamma_{0}$ is the strain amplitude and $\omega$ is the characteristic frequency. A convenient characterization of these rheology experiments is defined through the Deborah number 
$\mathrm{De}=\omega \lambda$ and the Weissenberg number $\mathrm{Wi} \equiv \lambda \gamma_{0}$, where $\lambda$ is the characteristic relaxation time of the material-in simple words, these two numbers determine how fast and how strong we are probing the viscoelastic system. Small Wi and large De corresponds to linear elasticity, in which the stress output is linearly dependent on the external input.

Generally speaking, we can draw the so-called Pipkin diagram [16], picturing the phase space of the system in function of the values of these two numbers. In the largest region of this diagram, strain amplitudes are large and frequencies are neither high nor low; experiments probing that region are called LAOS tests $[17,18]$ and are the subject of this Letter.

In the LAOS regime, linear viscoelasticity is not applicable anymore; the response is fully nonlinear, the storage and loss moduli become nontrivial functions of the strain amplitude $\gamma_{0}$. Very little is known in this regime, quoting Pipkin himself: "Here Be Dragons" [16].

From a totally different perspective, in the last ten years, holography revealed to be a very useful tool for the development and understanding of hydrodynamics [1924]. The most famous examples are (i) the formulation of a universal bound on the viscosity-to-entropy ratio [25] which is so far respected by all known fluids [26]; (ii) the discovery of new transport coefficients in anomalous hydrodynamics $[27,28]$ experimentally observed in Weyl semimetals [29].

A fundamental breakthrough in this direction is the observation that black holes (BHs) behave as dissipative hydrodynamic systems [30,31]. From this point of view, the application of an external strain source to the hydrodynamic system corresponds to the perturbation of the $\mathrm{BH}$ geometry by dynamical gravitational waves [19].

More recently, a series of works [32-35] explained how to endow black holes with a solid structure, providing them with a finite elastic response. In these new holographic theories, the $\mathrm{BH}$ response is no longer purely hydrodynamic but it becomes viscoelastic [36] in all aspects. Since then, a lot of effort has been devoted to the implementation, the classification and the characterization of these setups and similar ones [37-48]. In this Letter, we provide the first characterization of the nonlinear and time dependent viscoelastic response of these holographic models, with particular emphasis on the LAOS regime. The relevance of our results is diverse and highly interdisciplinary: (i) to shed light on the challenge of LAOS and in particular the physics of complex fluids (yelding, shear thinning, stress overshoot, dynamical instabilities) [18]; (ii) to reach a full characterization and understanding of the homogeneous holographic models with broken translations [32,49-51] and their possible connections with glasses, complex fluids and amorphous systems [52]; (iii) to study out-of-equilibrium processes in strongly coupled field theories and the possible universal evolution after dynamical quenches. Similar studies have been performed in Ref. [53], for a
CFT driven by an oscillating composite scalar operator, and [54] where a gapped holographic system has been perturbed with a homogeneous gravitational periodic driving. Some qualitative features observed in Ref. [53] are totally consistent with our findings.

The holographic model.-We consider a four-dimensional holographic massive gravity model $[32,33]$ defined by the following action:

$$
S=M_{p}^{2} \int d^{4} x \sqrt{-g}\left[\frac{R}{2}+\frac{3}{\ell^{2}}-m^{2} V(X)\right],
$$

with $X \equiv \frac{1}{2} g^{\mu \nu} \partial_{\mu} \phi^{I} \partial_{\nu} \phi^{I}$. The Stückelberg fields admit a radially constant profile $\phi^{I}=x^{I}$, which breaks the translational invariance of the dual field theory. For the rest of the Letter, we focus on the specific potential $V(X)=X^{3}$, which realizes the spontaneous symmetry breaking of translations and gives rise to a finite elastic response in the dual field theory and to the presence of propagating phonon modes - the corresponding Goldstones [35,39,55,56]. [See Refs. [34,38,40-42] for different choices of the potential $V(X)$ and the corresponding dual field theory properties.] For the potential considered in this work, the sound speeds of transverse and longitudinal phonons are subluminal $[35,39,56]$. Moreover, the elastic response is accompanied by a viscous dissipative contribution [37], which qualifies the model as viscoelastic [36]. [In our model, the ISO(2) global symmetry, typical of solids [10], is not gauged in the bulk. It would be nice to understand better the physical consequences of this fact. For an elegant solution to this issue, see Ref. [47].] One direct consequence of the competition between elasticity and dissipation is the observation of a sound to diffusion crossover in the spectrum of transverse phonons [40], analogous to the Ioffe-Regel crossover in dissipative systems [57].

In the linear regime-valid when the external deformations are small-we can use linear response theory to obtain the shear correlator from the bulk theory using the holographic dictionary. In the limit of zero momentum, the stress tensor correlator reads

$$
\mathcal{G}_{T_{x y} T_{x y}}^{R}(\omega, k=0) \equiv G^{\prime}(\omega)+i G^{\prime \prime}(\omega),
$$

and it defines for us the storage modulus $G^{\prime}(\omega)$ and the loss modulus $G^{\prime \prime}(\omega)$, together with the loss angle (phase shift) $\tan \delta(\omega) \equiv G^{\prime \prime}(\omega) / G^{\prime}(\omega)$. At low frequency we have,

$$
\mathcal{G}_{T_{x y} T_{x y}}^{R}(\omega, k=0)=G_{0}-i \eta \omega+\mathcal{O}\left(\omega^{2}\right),
$$

where $G_{0}$ and $\eta$ are the static shear modulus and the shear viscosity, respectively.

In a perfect elastic solid, we have $G^{\prime \prime}=0$ and $\delta=0$, while in a purely dissipative fluid $G^{\prime}=0$ and $\delta=\pi / 2$. All the materials with $0<\delta<\pi / 2$ are by definition viscoelastic. 
In the holographic model considered, at $m=0$, the static elastic modulus is null, $G_{0}=0$, and the system is a dissipative viscous fluid [saturating the Kovtun-Son-Starinets (KSS) bound, $\eta / s=1 / 4 \pi[25]$ ]. At intermediate and finite $m / T$, the system has both a finite static modulus and a finite viscosity and it displays viscoelastic properties - for details see the Supplemental Material [58]. The larger the parameter $m$-the mass of the graviton-the stronger the elastic component.

Nonlinear rheology.-Whenever the amplitude of the applied strain is large, nonlinearities set in and the linear viscoelastic approximation fails. From a gravitational point of view, this problem requires a more complicated timedependent setup which is explained in detail in the Supplemental Material [58], following the seminal work of Ref. [59]. (Temperature is not a well-defined concept out of equilibrium [60]. We will indicate with the symbol $T_{\text {in }}$ the temperature of the initial equilibrium state.) Within this regime, the produced stress is no longer linearly proportional to the applied strain but it presents a distorted shape which can be understood as a superposition of different Fourier components. More specifically, in the nonlinear regime, the strain $\gamma$ and the stress $\sigma$ can be represented as (The reason why only odd powers appear in the expansion is that the stress response is typically taken independent of the shear direction.)

$\gamma(t)=\gamma_{0} \sin (2 \pi \omega t), \quad \dot{\gamma}(t)=2 \pi \omega \gamma_{0} \cos (2 \pi \omega t)$,

$\sigma(t)=\sum_{p, \text { odd } q, \text { odd }} \sum_{0}^{p}\left[a_{p q} \sin (2 \pi q \omega t)+b_{p q} \cos (2 \pi q \omega t)\right]$

where $a_{11}, b_{11}$ correspond to the complex moduli $G^{\prime}(\omega)$, $G^{\prime \prime}(\omega)$ in the linear regime, and the first nonlinear corrections entering at order $\mathcal{O}\left(\gamma_{0}^{3}\right)$.

In this Letter, we will explore different methods to represent and characterize the nonlinear response at large amplitudes: (i) the analysis of the Fourier spectrum of the time dependent stress response, (ii) the Lissajous figuresstress-strain parametric curves $\{\gamma(t), \sigma(t)\}$, and (iii) the definition of the nonlinear complex moduli and their dependence on the strain amplitude.

First, we observe in Fig. 1 that by increasing the amplitude of the applied strain the shape of the stress response gets distorted and it deviates from a simple oscillatory function. This behavior is also displayed in the corresponding Lissajous figures which are no longer a simple oval, as expected in the linear regime. We notice that the shape of the curve after each cycle appears to be slightly modified; this phenomenon emphasizes the complexity of our viscoelastic system.

In Fig. 2 we study the Fourier spectrum of the signal. At small amplitude (orange curve), the spectrum is localized on the first and only harmonic, which is fixed by the frequency of the applied strain signal. This means the

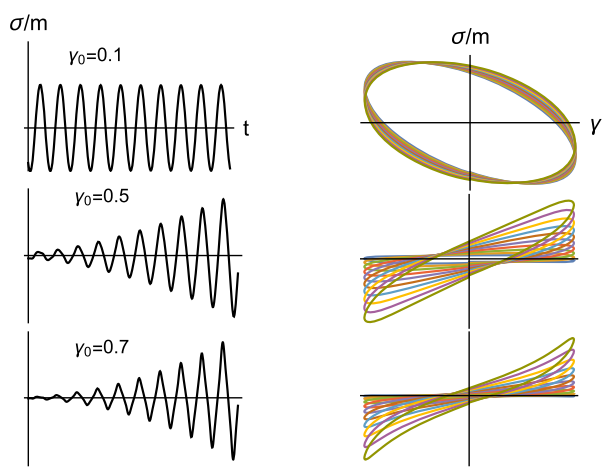

FIG. 1. The onset of nonlinear elasticity by increasing the strain amplitude. The strain is $\gamma(t)=\gamma_{0} \sin (2 \pi \omega t)$ with a smooth growing amplitude. Each color in the Lissajous figures corresponds to the $i$ th period. We fix $m / T_{\text {in }}=1.81, \omega / m=0.32$.

system is still in the linear response regime, where the stress is linearly proportional to the applied strain. By increasing the amplitude, higher (odd) harmonics appear in the spectrum confirming the functional structure displayed in Eq. (6). The normalized power of the higher harmonics, $I_{n / 1}$ is shown in Fig. 3. We find preliminary evidence for a power-law behavior $\sim \gamma_{0}^{n}$, which was previously suggested by theoretical arguments in Ref. [61].

Continuing along the lines of Eq. (6), we can rewrite the stress response as

$\sigma(t)=\sum_{n, \text { odd }} \sum_{m, \text { odd }}^{n} \gamma_{0}^{n}\left[G_{n m}^{\prime} \sin (2 \pi m \omega t)+G_{n m}^{\prime \prime} \cos (2 \pi m \omega t)\right]$.

The complex moduli are rigorously defined only in the linear regime; however, the measurements of $G^{\prime}\left(\gamma_{0}\right)$ and $G^{\prime \prime}\left(\gamma_{0}\right)$ at a fixed frequency can provide meaningful information. The most common option to calculate the moduli from a nonsinusoidal response consists in looking at the quantities $G_{1}^{\prime}\left(\omega, \gamma_{0}\right), G_{1}^{\prime \prime}\left(\omega, \gamma_{0}\right)$, defined as the

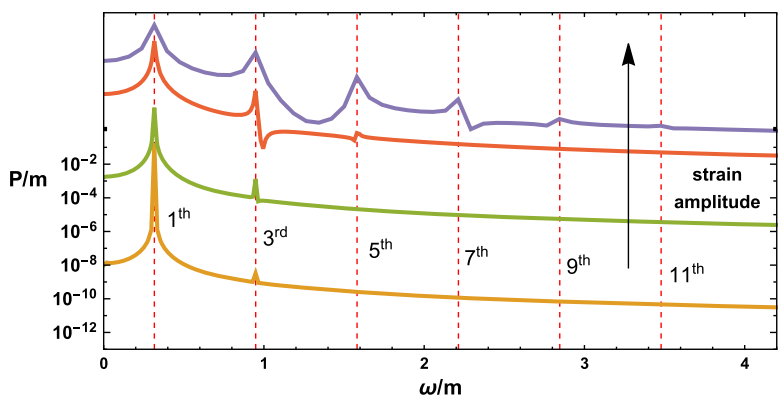

FIG. 2. Fourier spectrum $\mathcal{P}$ of the time dependent stress for increasing strain amplitude $\gamma_{0}=\{0.01,0.1,0.4,0.75\}$ (from orange to blue). Increasing the strain amplitude higher (odd) harmonics appear. The power spectrum is defined as $\mathcal{P}(\omega) \equiv$ $\mathcal{F}\left[\int_{-\infty}^{\infty} \sigma(t+\tau) \sigma(t) d t\right]$ 


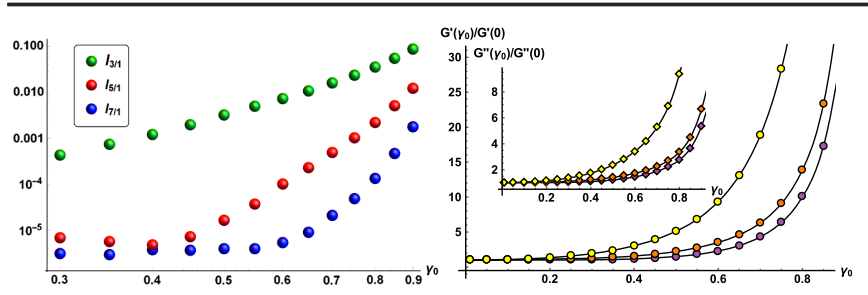

FIG. 3. Left: The normalized intensity $I_{n / 1} \equiv P\left(\omega_{n}\right) / P\left(\omega_{1}\right)$ of the first three higher harmonics in function of the strain amplitude $\gamma_{0}$. Right: The first complex moduli $G_{1}^{\prime}\left(\omega, \gamma_{0}\right), G_{1}^{\prime \prime}\left(\omega, \gamma_{0}\right)$ at fixed frequency, in function of the strain amplitude. We normalized them by their linear value $G(0) \equiv G\left(\gamma_{0} \ll 1\right)$. The colors correspond to $m / T_{\text {in }}=0.01,1.81,30$ (from yellow to purple). The onset of nonlinearity is roughly independent of the value of $m / T_{\text {in }}$ and it appears around $\gamma_{0} \sim 0.3$.

contributions from the first harmonics $\sin (2 \pi \omega t)$, $\cos (2 \pi \omega t)$ to the expansion in Eq. (7). Additionally, the values of $G_{1}^{\prime}, G_{1}^{\prime \prime}$ are exactly what the commercial rheometers provide in the experiments. Using a simple expansion, we obtain the first term in the sum of Eq. (7):

$$
\begin{aligned}
\sigma(t)^{\text {firstterm }} & =\left[G_{11}^{\prime} \gamma_{0}+G_{31}^{\prime} \gamma_{0}^{3}+\mathcal{O}\left(\gamma_{0}^{5}\right)\right] \sin (2 \pi \omega t) \\
& +\left[G_{11}^{\prime \prime} \gamma_{0}+G_{31}^{\prime \prime} \gamma_{0}^{3}+\mathcal{O}\left(\gamma_{0}^{5}\right)\right] \cos (2 \pi \omega t) \\
& =G_{1}^{\prime}\left(\omega, \gamma_{0}\right) \gamma_{0} \sin (2 \pi \omega t)+G_{1}^{\prime \prime}\left(\omega, \gamma_{0}\right) \gamma_{0} \cos (2 \pi \omega t),
\end{aligned}
$$

where we are neglecting the higher harmonics corrections which naturally appear in Eq. (7). Given these notations, the values of $G_{1}^{\prime}, G_{1}^{\prime \prime}$ at zero strain correspond to the linear response limit in Eq. (3). We plot the dependence of the first nonlinear complex moduli $G_{1}^{\prime}\left(\omega, \gamma_{0}\right), G_{1}^{\prime \prime}\left(\omega, \gamma_{0}\right)$ at fixed frequency in Fig. 3. We observe that for small amplitudes the moduli are independent of the strain amplitude. This is not true anymore at large amplitudes where nonlinear effects become important. We find that the onset of nonlinearity is roughly independent of the value of $m / T_{\text {in }}$ and it depends solely on the amplitude of the applied strain $\gamma_{0}$. Notice that in the nonlinear regime both moduli grow in a faster-than-linear fashion. From an operational point of view, this defines the presence of strain stiffening. This behavior is typical of hyperelastic materials such as rubberlike systems or complex polymers and it is in contrast to the so-called strain hardening, which is on the contrary a common feature of rigid metals.

Let us also notice that at low strain, for small values of the mass $m, G_{1}^{\prime \prime}>G_{1}^{\prime}$, indicating that our dual field theory is a viscoelastic liquid. This is reversed at large values of $m / T_{\text {in }}$, where the system becomes a viscoelastic solid with $G_{1}^{\prime \prime}<G_{1}^{\prime}$ [62] [see Fig. (A.2) in the Supplemental Material [58] ]. This is totally consistent with the fact that the graviton mass $m$ determines the "amount of solidity" of the system-its rigidity.

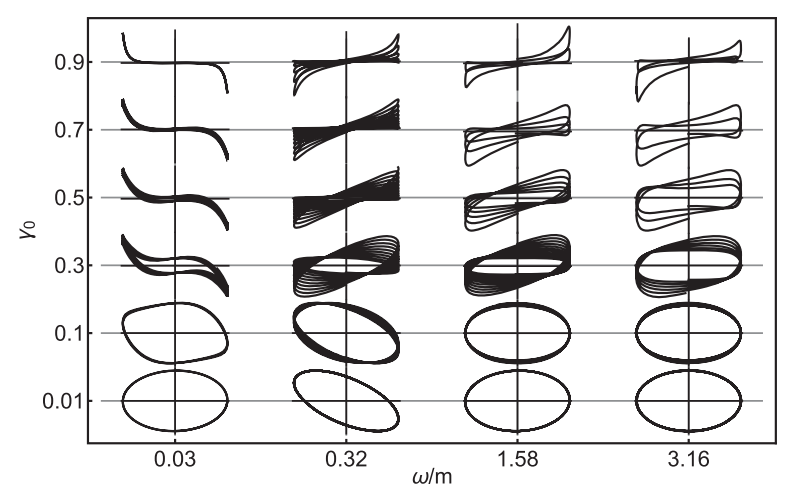

FIG. 4. Pipkin diagram: The Lissajous figures in function of the amplitude and the frequency of the oscillatory strain source. For this plot we fix $m / T_{\text {in }}=1.81$. This choice relates to a regime where our system is concretely viscoelastic.

A second possibility to characterize the nonlinear response, which is explored in detail in the Supplemental Material [58], consists of defining the complex moduli from the Lissajous figures looking at the tangent and the secant of the curve. Using this second method, we consistently find that the small amplitude modulus is smaller than the large amplitude one [see Fig. (A.6) in the Supplemental Material [58]] confirming the strain stiffening scenario.

To complete our analysis, we construct the Pipkin diagram of our model in Fig. 4 by plotting the Lissajous figures at various strain frequencies and amplitudes. We observe a neat transition between a linear viscoelastic regime at low amplitude and frequency to a more complicated large regions where the response becomes highly nonlinear (notice the similarities with Ref. [53]). This last result confirms that the regime we investigated cannot be described by linear response and it displays all the main physical properties of LAOS systems.

Discussion.-In this Letter, we characterize the nonlinear and time dependent mechanical response of viscoelastic (strongly coupled) field theories using holographic techniques. We focus our analysis on oscillatory external strains and on the regime of LAOS. We prove that the viscoelastic response is quite similar to that of complex fluids and hyperelastic materials (e.g., rubber) and in particular it exhibits a very neat strain stiffening phenomenon (see Ref. [63] for some concrete experimental data), which suggests that the holographic models at hand are not suitable to describe ordered metallic crystals characterized by strain hardening. We also observe a transition between a viscoelastic liquid behavior at small graviton mass, $m / T \ll 1$, to a viscoelastic solid regime at large values, which confirms the identification of the graviton mass with the rigidity of the dual field theory.

This work opens a new path for the study of complex fluids and viscoelastic systems using the holographic methods, which so far have been successfully applied only to strongly coupled liquids with no elastic response. There 
are several direct and interesting directions to pursue. First, it would be desirable to reach a better theoretical understanding of our numerical data by comparing our results to known phenomenological models such as the multi-mode Giesekus model $[61,64]$.

Second, a more extensive exploration of the phase diagram and possibly an extension of the study to include also the Chebyshev analysis [65] are certainly needed to draw universal conclusions.

On a more phenomenological perspective, one could consider different types of experiments, i.e., different signals for the applied strain such as building up functions, step functions, and quenches $[66,67]$. This extension would permit the study of extremely interesting phenomena such as nonlinear relaxation, stress overshoot, yielding, which represent still open challenges for rheology and condensed matter in general. In this respect, the existence of possible universal (relaxation) timescales is certainly a fundamental question to answer.

Finally, another relevant question which our work poses is the possibility of having holographic models displaying strain hardening and being therefore more suitable to describe metallic solids. This nicely connects to a fundamental and still open question: which kind of solids do these holographic systems describe? As shown in this Letter, the analysis of nonlinear transport properties is certainly a way of resolving this conundrum.

We thank Martin Ammon, Alex Buchel, Victor C. Castillo, Romulad Janik, Andreas Karch, Marco Laurati, Javier Mas, Ayan Mukhopadhyay, Oriol Pujolas, Yuho Sakatani, Laurence Yaffe, and Alessio Zaccone for interesting discussions and useful comments about the topic of the manuscript and an early version of this draft. S. G. thanks Martin Ammon for the collaboration on a related project. M. B. acknowledges the support of the Spanish MINECO's "Centro de Excelencia Severo Ochoa" Programme under Grant No. SEV-2012-0249. S. G. gratefully acknowledges financial support by the DAAD (German Academic Exchange Service) for a Jahresstipendium für Doktorandinnen und Doktoranden (One-Year Research grant for doctoral candidates).

*matteo.baggioli@uam.es † sebastian.grieninger@gmail.com thesam@m.scnu.edu.cn

[1] L. Landau, E. Lifshitz, A. Kosevich, J. Sykes, L. Pitaevskii, and W. Reid, Theory of Elasticity, Course of Theoretical Physics (Elsevier Science, ButterworthHeinemann, 1986).

[2] L. Landau and E. Lifshitz, Fluid Mechanics (Elsevier Science, Pergamon Press, 2013), Vol. 6.

[3] R. Christensen, Theory of Viscoelasticity: An Introduction (Academic Press, New York, 1971).
[4] H. Barnes, K. J. F. Hutton, J. Hutton, and K. Walters, An Introduction to Rheology, Rheology Series (Elsevier Science, Amsterdam, 1989).

[5] R. G. Larson, The Structure and Rheology of Complex Fluids (Oxford University Press, New York, 1999), Vol. 150.

[6] D. Roylance, Engineering viscoelasticity (2001), http://ocw .mit.edu/courses/materials-science-and-engineering/3-11mechanics-of-materials-fall-1999/modules/visco.pdf.

[7] A. Malkin, A. Malkin, and A. Isayev, Rheology: Concepts, Methods, and Applications (ChemTec Pub., Toronto, 2006).

[8] A. Nicolis, R. Penco, F. Piazza, and R. Rattazzi, Zoology of condensed matter: Framids, ordinary stuff, extra-ordinary stuff, arXiv:1501.03845.

[9] S. Endlich, A. Nicolis, R. A. Porto, and J. Wang, Dissipation in the effective field theory for hydrodynamics: First order effects, Phys. Rev. D 88, 105001 (2013).

[10] A. Nicolis, R. Penco, F. Piazza, and R. Rattazzi, Zoology of condensed matter: Framids, ordinary stuff, extra-ordinary stuff, J. High Energy Phys. 06 (2015) 155.

[11] P. Kovtun, Lectures on hydrodynamic fluctuations in relativistic theories, J. Phys. A 45, 473001 (2012).

[12] H. Liu and P. Glorioso, Lectures on non-equilibrium effective field theories and fluctuating hydrodynamics, Proc. Sci. TASI2017 (2018) 008.

[13] F. Lockett, Nonlinear Viscoelastic Solids (Academic Press, London, New York, 1972).

[14] G. A. Holzapfel, Nonlinear solid mechanics: A continuum approach for engineering science, Meccanica 37, 489 (2002).

[15] S. Wang, Nonlinear Polymer Rheology (Wiley, Hoboken, 2018).

[16] A. Pipkin, Lectures on Viscoelasticity Theory, Applied mathematical sciences No. v. 7, pt. 1 (Springer-Verlag, New York, 1986).

[17] K. Hyun, M. Wilhelm, C. O. Klein, K. S. Cho, J. G. Nam, K. H. Ahn, S. J. Lee, R. H. Ewoldt, and G. H. McKinley, A review of nonlinear oscillatory shear tests: Analysis and application of large amplitude oscillatory shear (LAOS), Prog. Polym. Sci. 36, 1697 (2011).

[18] S. Rogers, Large amplitude oscillatory shear: Simple to describe, hard to interpret, Phys. Today 71, No. 7, 34 (2018).

[19] G. Policastro, D. T. Son, and A. O. Starinets, From AdS/ CFT correspondence to hydrodynamics, J. High Energy Phys. 09 (2002) 043.

[20] G. Policastro, D. T. Son, and A. O. Starinets, From AdS/ CFT correspondence to hydrodynamics. 2. Sound waves, J. High Energy Phys. 02 (2002) 054.

[21] R. A. Janik, Viscous Plasma Evolution from Gravity Using AdS/CFT, Phys. Rev. Lett. 98, 022302 (2007).

[22] P. K. Kovtun and A. O. Starinets, Quasinormal modes and holography, Phys. Rev. D 72, 086009 (2005).

[23] P. K. Kovtun, D. T. Son, and A. O. Starinets, Viscosity in Strongly Interacting Quantum Field Theories from Black Hole Physics, Phys. Rev. Lett. 94, 111601 (2005).

[24] P. Kovtun, D. T. Son, and A. O. Starinets, Holography and hydrodynamics: Diffusion on stretched horizons, J. High Energy Phys. 10 (2003) 064.

[25] G. Policastro, D. T. Son, and A. O. Starinets, The Shear Viscosity of Strongly Coupled $N=4$ Supersymmetric Yang-Mills Plasma, Phys. Rev. Lett. 87, 081601 (2001). 
[26] S. Cremonini, The Shear viscosity to entropy ratio: A status report, Mod. Phys. Lett. B 25, 1867 (2011).

[27] K. Landsteiner, E. Megias, and F. Pena-Benitez, Gravitational Anomaly and Transport, Phys. Rev. Lett. 107, 021601 (2011).

[28] K. Landsteiner, E. Megias, L. Melgar, and F. Pena-Benitez, Holographic gravitational anomaly and chiral vortical effect, J. High Energy Phys. 09 (2011) 121.

[29] J. Gooth et al., Experimental signatures of the mixed axialgravitational anomaly in the Weyl semimetal $\mathrm{NbP}$, Nature (London) 547, 324 (2017).

[30] K. S. Thorne, R. H. Price, and D. A. MacDonald, Black Holes: The Membrane Paradigm (Yale University Press, New Haven, 1986), p. 367.

[31] N. Iqbal and H. Liu, Universality of the hydrodynamic limit in AdS/CFT and the membrane paradigm, Phys. Rev. D 79, 025023 (2009).

[32] M. Baggioli and O. Pujolas, Electron-Phonon Interactions, Metal-Insulator Transitions, and Holographic Massive Gravity, Phys. Rev. Lett. 114, 251602 (2015).

[33] L. Alberte, M. Baggioli, A. Khmelnitsky, and O. Pujolas, Solid holography and massive gravity, J. High Energy Phys. 02 (2016) 114.

[34] L. Alberte, M. Ammon, M. Baggioli, A. Jiménez, and O. Pujolàs, Black hole elasticity and gapped transverse phonons in holography, J. High Energy Phys. 01 (2018) 129.

[35] L. Alberte, M. Ammon, M. Baggioli, A. Jiménez-Alba, and O. Pujolàs, Holographic Phonons, Phys. Rev. Lett. 120, 171602 (2018).

[36] T. Andrade, M. Baggioli, and O. Pujolàs, Viscoelastic dynamics in holography, Phys. Rev. D 100, 106014 (2019).

[37] L. Alberte, M. Baggioli, and O. Pujolas, Viscosity bound violation in holographic solids and the viscoelastic response, J. High Energy Phys. 07 (2016) 074.

[38] M. Baggioli and S. Grieninger, Zoology of solid \& fluid holography-Goldstone modes and phase relaxation, J. High Energy Phys. 10 (2019) 235.

[39] M. Ammon, M. Baggioli, S. Gray, and S. Grieninger, Longitudinal sound and diffusion in holographic massive gravity, J. High Energy Phys. 10 (2019) 064.

[40] M. Ammon, M. Baggioli, and A. Jiménez-Alba, A unified description of translational symmetry breaking in holography, J. High Energy Phys. 09 (2019) 124.

[41] M. Baggioli and K. Trachenko, Maxwell interpolation and close similarities between liquids and holographic models, Phys. Rev. D 99, 106002 (2019).

[42] M. Baggioli and K. Trachenko, Low frequency propagating shear waves in holographic liquids, J. High Energy Phys. 03 (2019) 093.

[43] M. Baggioli and A. Buchel, Holographic viscoelastic hydrodynamics, J. High Energy Phys. 03 (2019) 146.

[44] S. Grozdanov and N. Poovuttikul, Generalized global symmetries in states with dynamical defects: The case of the transverse sound in field theory and holography, Phys. Rev. D 97, 106005 (2018).

[45] A. Amoretti, D. Arean, B. Gouteraux, and D. Musso, Effective holographic theory of charge density waves, Phys. Rev. D 97, 086017 (2018).
[46] J. Armas and A. Jain, Viscoelastic hydrodynamics and holography, J. High Energy Phys. 01 (2020) 126.

[47] A. Esposito, S. Garcia-Saenz, A. Nicolis, and R. Penco, Conformal solids and holography, J. High Energy Phys. 12 (2017) 113.

[48] M. Fukuma and Y. Sakatani, Conformal higher-order viscoelastic fluid mechanics, J. High Energy Phys. 06 (2012) 102.

[49] T. Andrade and B. Withers, A simple holographic model of momentum relaxation, J. High Energy Phys. 05 (2014) 101.

[50] A. Donos and J. P. Gauntlett, Holographic Q-lattices, J. High Energy Phys. 04 (2014) 040.

[51] D. Vegh, Holography without translational symmetry, arXiv:1301.0537.

[52] D. Facoetti, G. Biroli, J. Kurchan, and D. R. Reichman, Classical glasses, black holes, and strange quantum liquids, Phys. Rev. B 100, 205108 (2019).

[53] M. Rangamani, M. Rozali, and A. Wong, Driven holographic CFTs, J. High Energy Phys. 04 (2015) 093.

[54] A. Biasi, J. Mas, and A. Serantes, Gravitational wave driving of a gapped holographic system, J. High Energy Phys. 05 (2019) 161.

[55] M. Baggioli, U. Gran, A. J. Alba, M. Tornsö, and T. Zingg, Holographic plasmon relaxation with and without broken translations, J. High Energy Phys. 09 (2019) 013.

[56] M. Baggioli, V. Cancer Castillo, and O. Pujolas, Scale invariant solids, arXiv:1910.05281.

[57] M. Baggioli and A. Zaccone, Universal Origin of Boson Peak Vibrational Anomalies in Ordered Crystals and in Amorphous Materials, Phys. Rev. Lett. 122, 145501 (2019).

[58] See Supplemental Material at http://link.aps.org/ supplemental/10.1103/PhysRevLett.124.081601 for the technicalities concerning the numerical routine and more details about the linear and non-linear viscoelastic response.

[59] P. M. Chesler and L. G. Yaffe, Numerical solution of gravitational dynamics in asymptotically anti-de Sitter spacetimes, J. High Energy Phys. 07 (2014) 086.

[60] A. Puglisi, A. Sarracino, and A. Vulpiani, Temperature in and out of equilibrium: A review of concepts, tools and attempts, Phys. Rep. 709, 1 (2017).

[61] R. B. Bird and J. M. Wiest, Constitutive equations for polymeric liquids, Annu. Rev. Fluid Mech. 27, 169 (1995).

[62] M. Laurati, P. Maßhoff, K. J. Mutch, S. U. Egelhaaf, and A. Zaccone, Long-lived neighbors determine the rheological response of glasses, Phys. Rev. Lett. 118, 018002 (2017).

[63] K. A. Erk, K. J. Henderson, and K. R. Shull, Strain stiffening in synthetic and biopolymer networks, Biomacromolecules 11, 1358 (2010).

[64] J. G. Nam, K. Hyun, K. H. Ahn, and S. J. Lee, Analysis of the normal stress differences of viscoelastic fluids under large amplitude oscillatory shear flow, AIP Conf. Proc. 1027, 171 (2008).

[65] K. Cho, Viscoelasticity of Polymers, Springer Series in Materials Science (Springer, Netherlands, 2018).

[66] M. Ammon, S. Grieninger, A. Jimenez-Alba, R. P. Macedo, and L. Melgar, Holographic quenches and anomalous transport, J. High Energy Phys. 09 (2016) 131.

[67] S. Grieninger, Holographic quenches and anomalous transport, arXiv:1711.08422. 